\title{
The BIM based Responsive Environmental Performance Design Methodology
}

> YONGHENG HU

University of Applied Arts Vienna, Austria

toto4356@gmail.com

> QINYING LI

University of Michigan, USA

liqinying@gmail.com
> FENG YUAN

Tongji University, China

phillipyuan0007@gmail.com

> HAN LI

Archi-Union Architects, China

hanlihanlihanl@gmail.com

\begin{abstract}
The concept of "families" lies in the core of internal data structure in Building Information Modeling (BIM). The elements of this modeling platform are all associated with each other as parts of the "families", independent of their geometrical structure, materiality, parametric dependencies or their physical connection to other elements. Through the associations introduced among the parameters of the 'families' members, this study aims at establishing a methodology for a multi-objective evaluation of the environmental performance of the building as an organism. The methodology is founded on a system of different values and weights attributed to the parameters of the families members which are adjusted and fine-tuned through a series of iterations, thus affecting the overall building performance towards an optimum goal. The performance evaluation method used in the "families" methodology is not limited to the individual assessment of the environmental performance objectives or to an integrated multi-objective weighting mechanism; as an overall evaluation platform it checks and balances the individual characteristics of the system not as static conclusive results but as dynamic criteria intended to guide the overall design and building process. The importance of this paper lies in the construction of a concrete methodological set of tools for the assessment of the environmental performance of the building. It will lead the way in independent research in the field of architectural design and the development of ecological thinking and building in China.
\end{abstract}

Keywords: BIM 'families'; Multi-Objective Generic Algorithm; Environmental Performance Simulation; Environmental Performance Assessment; Multi-Objective Environmental Performance Optimization

\section{Introduction}

\subsection{The research objectives}

"Families" is an essential part of BIM (Building Information Modeling) systems. For example, Autodesk $\mathrm{R}^{\oplus}$ Revit $^{\circledR}$ is a combination of various ways created through the "families of systems", "build a families" and "built-in families created". The "Families" play multiple roles of building components such as doors, windows, walls, and composite ecological epidermal information integration, classification and parametric definition. Design objects for building information modeling are stored in a unified database by information integration, and users can add data according to their needs. BIM also provides information for a variety of analytical implantation conditions, and strong support for full life-cycle management and adaptive responsive optimization of construction projects.

- BIM platform builds performance evaluation system that resolves uncertainty in decision-making of construction projects of great significance. The design process of construction, involving concept, program and construction, is full of uncertainty. The BIM platform intervention offers a full range of solutions to various stages in the form of three mathematical models. The uncertainty of Building Performance stems from thorny issues that need to be addressed in the construction phase. After completing most part of the work, building performance assessment tends to affect the progress of the project design. Therefore, the solution for the working methods lies in studying multi-objective environment, performance evaluation and intelligent processing of design facilities.

- The fusion of multi-objective environment, performance analysis and architectural form generation, has far-reaching implications for scientific development of eco-energy saving in China. Today, China is widely followed in ecological energy efficiency specifications and software. Isolated or passive use of environmental performance evaluation cannot dominate the generation and control of the form of design, but there is no way to refine the construction drawings with precise digital construction process. This study, aimed at analyzing the environmental performance, led to building the design by means of parametric design reasonable flow, and thus to executing the design process more scientifically.

- In terms of the design method, the evaluation system of checks and balances requires establishment of the parameters of an integrated multi-objective performance. Such an evaluation will be an organic process of dynamic change, such as ventilation, thermal insulation and energy saving, which brings the day-lighting performance tuning and other indicators into synchronized display, and a comprehensive evaluation of the global view helps in 
determining a more balanced and effective optimization direction and approach.

- Performance evaluation system of multi-objective environment is of great significance to guide the design process of optimization. The evaluation results will become the basic parameters of optimization; multiconstraint optimization will drive through the intelligent algorithm BIM model "Families" data item adjustments related property, which itself is optimized and the results of the overall design performance drive "Families" improved.

To establish such a binary system of assessment and to optimize the expansion and extension of the traditional design ideas, the design method, based on the performance of the environment built from a simple single-target directional design, can be transformed into a multiquantitative design, which can carry out even real-time assessment of the progress of a number of environmental performance indicators. The optimal path allows designers to keep abreast of the design results of many environmental performance indicators and to make real-time adjustment of the design development achievements.

\subsection{The current exploration and research in industry}

For studying environmental performance of buildings, one needs to understand the ecological character of the building. In earlier studies, researchers followed some measures for improvement of environmental performance; this is the beginning of the performance optimization of the built environment. Similarly, the emergence of BIM "families" provides a full life-cycle study to optimize the environmental performance of buildings, from a single stage to the whole, and from individual target optimization to multi-objective optimization of collaborative work platform. In addition, system software is being constantly evaluated to optimize environmental performance of buildings.

Depending on the focus, the development work of building environmental performance can be divided into three stages: environmental performance "analog", which forms the basis of the entire study; environmental performance based simulation study; environmental performance "optimization", the ultimate purpose of this study. At present, the main research abroad is in the third stage. The major part of the second stage research, excluding some simple non-systematic research, such as "headache medicine head" class single-objective optimization, is not well established yet.

At the moment, for environmental performance of building comfort, the focus at home and abroad is usually on single-purpose simulation software to evaluate energy saving optimization and then offer some suggestions for improvement. As regards thermal performance, air conditioning load and thermal comfort software tools are many and varied. The accurate and widely used one in Scotland, UK is that of inte- grated environmental solutions ${ }^{\circledR}$ Co., Ltd. development IES ${ }^{\circledast}$ (Integrated Environ- mental Solutions Ltd). For wind environment performance, CFD simulation has be- come a fluid thermal analysis tool (e.g., StarCD ${ }^{\oplus}$, Steam ${ }^{\oplus}$, and other simpler com- mercial software). For lighting performance, Radiance ${ }^{\oplus}$ is an important means to analyze the natural light environment. For energy efficiency, the industry currently counts on the overall energy consumption of the building analytical evaluation. The most widely used software for this purpose, such as DOE2 ${ }^{\circledR}$, EnergyPlus ${ }^{\circledast}$, was de- veloped by the U.S. Department of Energy. Internationally, the software for architec- tural design multi-objective optimization, such as modeFRONTIER ${ }^{\oplus}$, Optimus ${ }^{\oplus}$, iSight ${ }^{\oplus}$, MATLAB $^{\circledR}$, etc. are popular. However, for multi-objective performance algorithm integrated optimization, the compromise conditions between the quantita- tive evaluation of each single target and single target is not a simple, automated pro- cess. Even so, there is no unified way to optimize the overall performance of the building.

However, the following are the academic problems encountered in international and domestic studies:

- The majority of the studies relate to single goal performance analysis and on systematic multi-objective performance analysis.

- What is widely applied in environmental performance of multi-objective optimization methods is mostly single-target performance analysis "overlay" on system- atic multi-objective performance analysis.

- Most software research studies on climate, environment and norms do not converge to facilitate analysis and rational review of the problems to be solved.

- The multi-objective performance analysis software is strong in assessing, but weak in optimizing the basis for feedback.

- It is doubtful if the optimized result can be achieved with the dynamic parameters of the design process evaluation and optimization of feedback.

- There are not many "performance evaluations" to "shape optimization" binary mode linkage. Also it is doubtful if the analysis of performance parameters by families of BIM models could help in assigning a new definition to architectural design process and in facilitating design innovation.

International and domestic researchers agree that multi-objective performance op- timization has become an effective way for solving this kind of problem. The qualita- tive interpretation of the concept is shown in Fig.1:

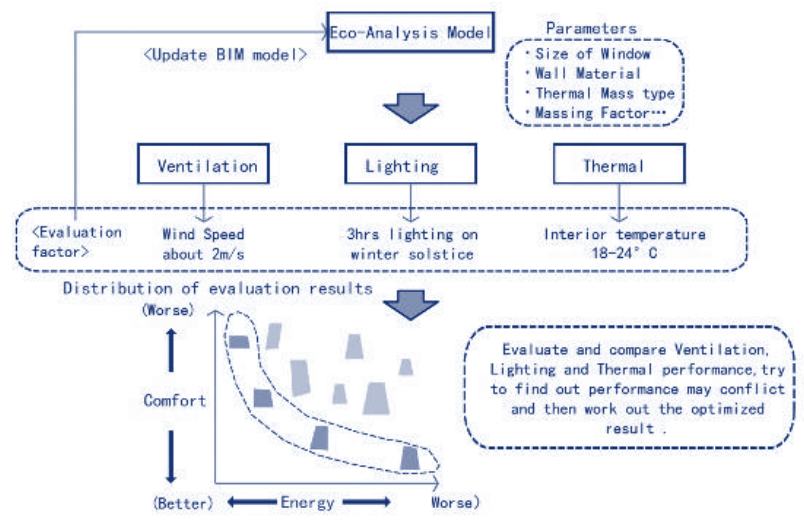

Fig. 1: Multi-objective environmental performance optimization design concept. 


\section{Project research BIM and parametric definition of "Families"}

\subsection{Studying the BIM "Families" geometric} properties and building environmental performance attributes of associated parameters.

"Families", the chief component of the BIM system, is an important link between the topics from the "performance evaluation" to "shape optimization" dual mode. "Families" not only saves on 2D and 3D modeling, CAD drawings, and between the components topology information, it can also accommodate the properties of the ob- ject information (such as windows "families", including dimensions, open fan size, type of glass, type of window frames, hardware and a lot of other information), and can provide seamless information to the performance analysis platform. And, more importantly, "Families" parameters define that "population" is not just a simple in-formation memory; it can also receive information, it can self-adjust, and it can re- spond to different driving conditions of the computing unit (See Fig.2).

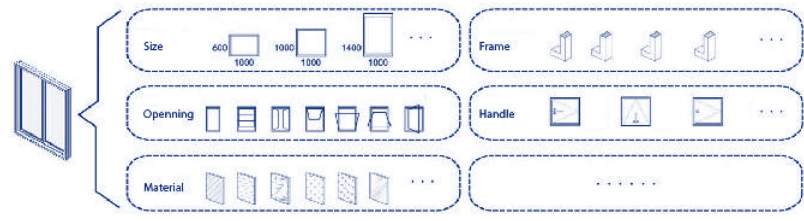

Fig. 2: Families object as a storage of parameters.

The key to designing the overall optimization is to break down optimization into various "families", so that they can improve the environmental performance of the entire architectural design. Simply put, if the evaluation results show that the architec- tural design of natural ventilation performance is poor, then bound to the size of the fan by changing the windows open, open the way to improve. But, as the computer cannot judge even a simple design logic, the correction must be achieved through the definition of the environmental performance of buildings associated with "families" parameter (See Fig.3).
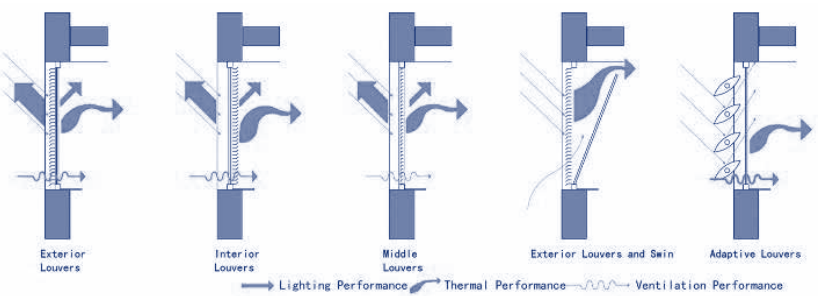

Fig. 3: Windows "families" with different louver parameters, variable environmental perfor- mance and a complex association in-between.

\section{Dynamic information exchange method and multi-objective environmental simulation}

\subsection{Digitized analog of the environmental performance of} buildings, evaluation methods and its Semantic Transformation. The goal is a step-by-step process of deepening the analysis of environmental per-formance in different architectural design stages, because the depth of the BIM mod- els will have to provide the information content. In the preliminary design stage, as BIM models provide building size, height, area and so on, the evaluation tends to be in a macro form, such as weather information, orientation, passive strategies and building volume; in the design development stage, as BIM models provide basic ar- chitectural model elements and the overall system together with some non-geometric information, the analysis will be concentrated on the sunshine, shade, thermal per-formance, ventilation and basic energy consumption; in the final construction design stage, because the BIM "families" elements to achieve a precise number, size, shape, materials, and analysis of information related to deepen the analysis of lighting, venti- lation, thermal calculation and energy consumption can be achieved very detailed report. This means that in the design phase, our evaluation objectives will be very different (Figure 4).

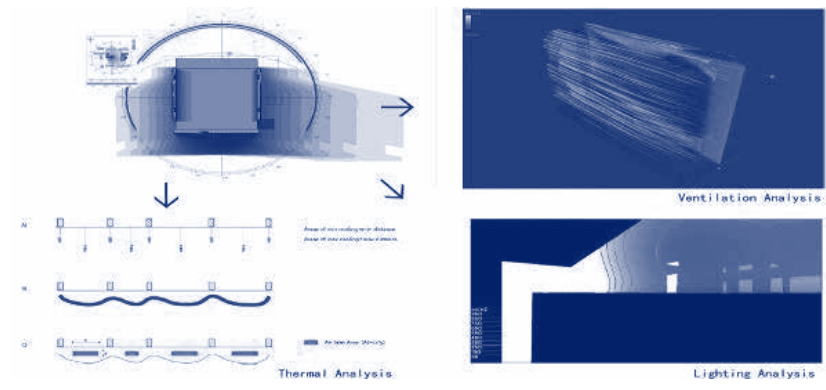

Fig. 4: Multi-objective performance evaluation.

Differentiation of the evaluation target enables simulation and environmental per- formance evaluation of uncertainty analysis platform and industry platform resources, but there is great difference between the scope of application and evaluation of the object between the platforms. This band between semantic analysis software and BIM software related problem is not a simple "one to one", but a multi-platform sharing one, which requires that multi-objective optimization process based BIM "families" environment Performance design method must be a cross-platform work. Usually, the evaluation of environmental performance of buildings is a comprehen- sive and weighty process. Keeping in view both technical and practical requirements, this study focused on three environmental aspects: natural light, natural ventilation and thermal performance. The evaluation methods consider two aspects: Indicator items and index weights (See Figure 5). Indicator item ensures to minimize the num- ber of indicators, simulation requirements and difficult parts of evaluation, although they weaken the actual optimization that can be implemented. At the same time, the selected indicators are generally accepted in the industry, thereby enhancing the cred-ibility of the assessment system. The index weight determination has been a difficult part of the evaluation. For this study, Delphi method was attempted with analytic hierarchy process combination and open weight coefficient interface, so that the eval- uation can cope with different external environmental conditions and obtain a higher sensitivity rating for the building performance requirements. 


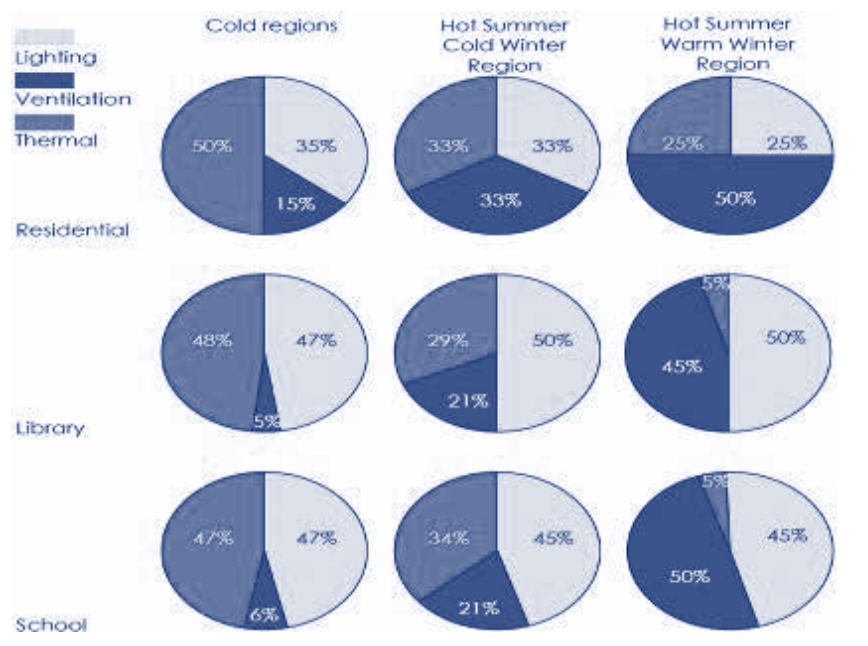

Fig. 5: Environmental performance according to different climatic conditions and functional requirements.

Design model is not exactly the same semantic exchange platform for BIM, "eval- uation" model, and data exchange in order to make the design model contains the information analysis platform read. One needs to consider the exchange of data be- tween different platforms; gbXML was selected as the basic data exchange format for the package "families" parameter finishing output. The gbXML data packet includes the following: 1 . Basic spatial data; 2 building envelope elements (walls, floor, ceiling) for analysis; 3 Forms hole element; 4. Other members used for the analysis elements. These data, with the deepening of the design phase, constantly enrich the data exchange that children need to refine regulation (Fig.6).

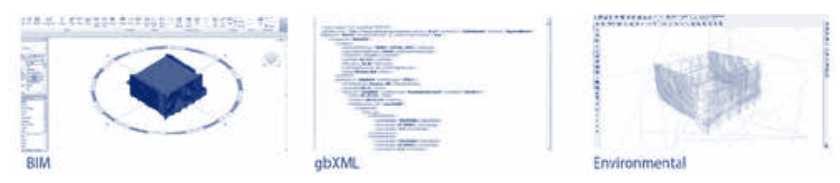

Fig. 6: BIM platform data exchange.

\subsection{The Adaptive Multi-objective Environmental}

\section{Performance Evaluation and Optimization}

In architectural design and environmental performance optimization process, most of the design and decision-making problems are multi-objective with different goals and hence they are likely to be mutually competitive; consequently, one cannot achieve simultaneous optimization of all the targets. For example, to increase the lighting of the room, if one increases the dimensions of the window glass, then it will inevitably bring about a decline in the performance of wall insulation. To ensure re- tention of the thermal insulation properties, one needs to increase the thermal insula- tion coefficient of the glass material also. Multi-objective optimization of environ- mental performance, in terms of the nature of the problem, does not mean a single global optimal solution, but evaluation indicators relating to an optimization solution set. To achieve the best optimization results through a multi-objective genetic algo- rithm optimization, one needs to carry out step-by-step optimization and screening (Figure 7). Multi-objective optimization genetic algorithm Life Science and Engineer- ing Science cross each other. Multi-objective genetic algorithm, a problem-solving global search algorithm, has become, in recent years, a hot research field of applied mathematics, information science, computer science, and many other subjects of common interest.

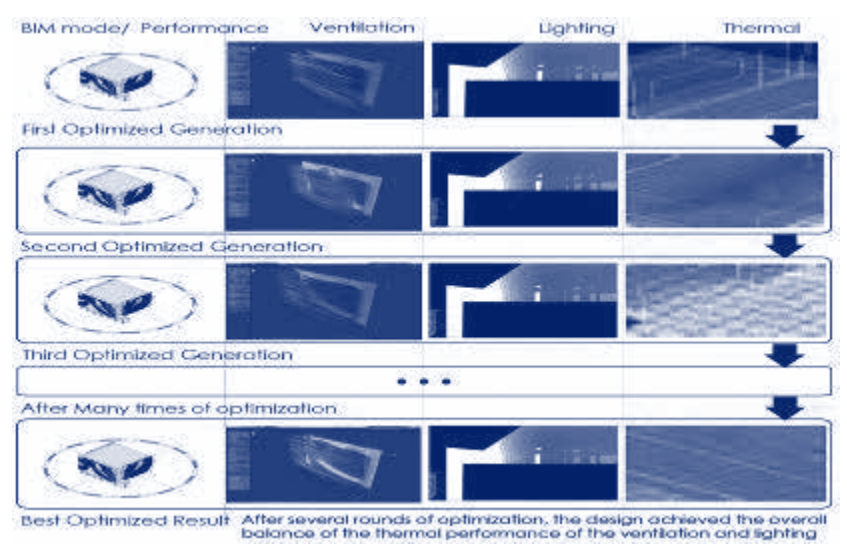

Fig. 7: Applying genetic algorithm to solve architectural design of environmental performance of multi-objective optimization problem.

Natural environment, like the evolution of life, will be selected based on the "fami- lies" and constantly pushes forward multi-objective environment results of the evalua- tion towards optimizing the environmental performance of buildings. "Families" pa- rameter iteration result is a dynamic solution set; this solution assembly will have to be evaluated by many environmental performance evaluations of the target platform. Further, it has to choose optimal results for building environment performance opti- mization tool (see Figure 8).

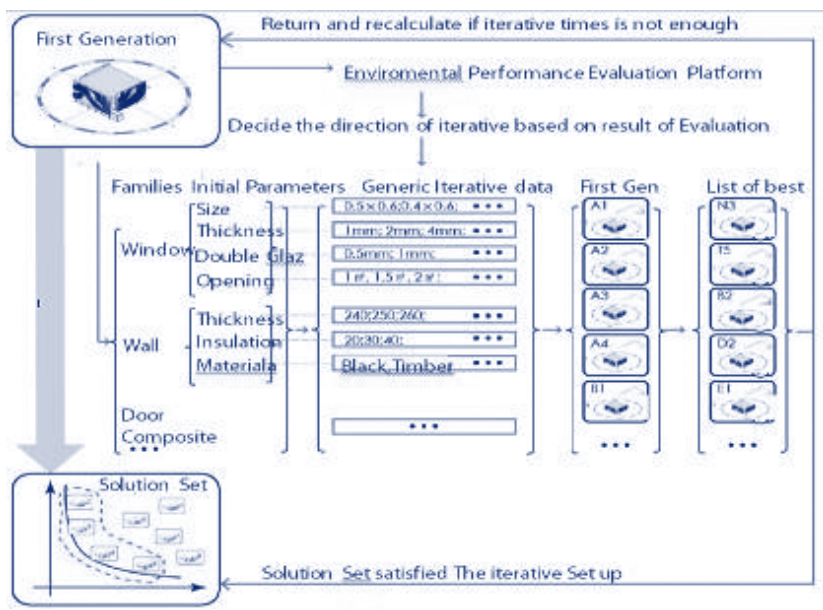

Fig. 8: Multi-objective environmental performance of iterative optimization graphic.

\section{Reflection and Conclusion}

4.1 Reflection and key scientific problems to be solved This study attempts to establish the parametric linkage mechanism between "multi- objective performance analysis" and "families". 
Owing to the availability of multiple inter-related software, evaluation of the feedback on multi-objective optimization method requires resolving of several key scientific issues.

- Scientific definition of BIM "families", and controlling the creation and modifi- cation process, from "Performance Evaluation" to "shape optimization" of binary linkage model are the key issues of this study.

The "families" definition starts with the creation of a parameterization process. For performance evaluation of multi-objective environment and a complete assessment of objects and data support, what is first required are the "families" information integra- tion properties and "families" parameter-driven attributes. This definition is deviates from geometry and topology-driven definition involving object's class, location and interface between semantic drive to bring more performance (structural performance, environmental performance and construction performance)

- In the context of the availability of different software, how the environmental performance parameters interact with BIM "families parameters to achieve a rea- sonable semantic association (SEM) is another scientific question.

BIM collaboration platform IFC (Industrial Foundation Classes) standard is an at- tempt to solve the semantic association (Semantic Exchange Modules) carrier. The BIM system tries to build architecture, engineering, performance analysis, construction management, equipment and logistics, and the construction process as well as the later part of property management and other aspects of the 3D-visualization of collab- orative work platform. This collaborative process must address the important issues of the accessibility of information input and output, because there is a big difference between various software packages and their ways of working; however, smooth se- mantic association has yet to be resolved. Environmental performance analysis soft- ware, such as Ecotect, or the conversion model Vesari BIM families model is not smooth; it does not translate directly between Maya, Rhino model and performance simulation. This subject must be studied through a variety of performance analyses and design software for establishing reasonable file format exchange standards and mutually agreeable conversion conditions.

- How to apply multi-objective genetic algorithm to optimize the analysis process, is another core scientific issue.

In recent years, multi-objective genetic algorithm has become a hot research field in computer science, information science and applied mathematics and many other subjects of common interest. This approach is used mainly in a variety of conditions to study how to make trade-offs, and balanced and comprehensive scientific deci-sions. The focus of this study is to understand how reasonable research methods are used in the performance of multi-objective optimization". The design process is not only a continuum embodying different functions - how to define the performance parameters of the multi-objective, how to analyze and determine the function set, and how to seek optimal solution for reasonable information feedback--it is also a dynam- ic scientific process. The core issue of this research problem is challenging, but it has strong research and application values.

\subsection{Conclusion of research and innovation}

- Features of BIM collaborative design platform, from "performance evaluation" to "shape optimization design method, are precise and dynamic.

The biggest breakthrough of this study is establishing the environmental perfor- mance of multi-objective optimization genetic algorithm model and feeding the eval- uation results directly to the optimization of the design. Of course, the realization of this goal cannot be separated from BIM platform "families" information integration attributes and parameter-driven properties, as also the development of the environ- mental assessment simulation platform; but, the most important thing is achieving the architectural design methodology based on the environmental performance of this goal. The outcome of present study suggests that the research be extended beyond the traditional design ideas. This is the dynamic feedback mechanism for construction body design innovation.

- "Families", as the starting point of this study, is to establish the environmental performance of the building geometry attribute parameters associated innovation.

Based on multi-objective environmental performance evaluation, optimization of the design of the system is yet to be established. The fundamental reason is though there are various analog evaluation platforms, the BIM platform does not interface with any information model to optimize the design results based on the evaluation index system theory. However, the emergence of "families", change this situation radically. Two special attributes of the "families" enable completion of the optimiza- tion process in an almost synchronized form: The first attribute is that "families" can carry out integrated property performance evaluation of multi-objective environment to provide a complete assessment of objects and data support; the second is that "fam-ilies" has the parameter-driven attribute; so along with the completion of the assess- ment, the direction of the index optimization, optimization algorithm to "families parameter corresponding to the input end to be driven to drive "families" multi- objective optimization driven by the optimization of the results of the overall design.

- Application of "Multi-objective genetic algorithm to environmental performance optimization analysis" is another feature of this study.

Ontology-oriented fundamental research in China is particularly relevant in this context. The author believes that absorption of interdisciplinary advanced methods will allow multi-objective genetic algorithm optimization analysis used in environ- mental performance, and thus act as a major catalyst in the evolution of design meth- odology. The multi-objective performance parameters are defined. Through analysis and functional optimization, together with scientific feedback, the analysis of envi- ronmental performance in the architectural design of the application can be taken to new heights.

\section{References}

[1] Daniel Davis, Brady Peters. Design Ecosystems: Customizing the Design Envi- ronment with plugins [J] .Architectural Design (forthcoming), 2013. 
[2] EhsanAsadi, Manuel Gameiroda Silva, Carlos HenggelerAntunes et al. Multi- objective optimization for buildingretrofit strategies:A model and an application [J].Energy andbuildings, 2012,44(Jan.):81-87.

[3] Ralph Evins, Philip Pointer, Ravi Vaidyanathan et al. A case study exploring regulated energy use in domestic buildings using design- of-experiments and multi- objective optimization [J]. Building andenvironment,2012,54(Aug.): 126-136.

[4] M. Fesanghary, S. Asadi, Zong Woo Geem et al. Design of low-emission and energy-efficient residential buildingsusing amulti-objective optimization algo- rithm[J].Building and environment,2012,47(Mar.):245-250.

[5] EhsanAsadi,ManuelGameiro da Silva,CarlosHenggelerAntunes et al. A multi- objective optimization model for building retrofit strategies using TRNSYS simula- tions, GenOpt and MATLAB[J].Building andenvironment, 2012,56(Oct.):370-378.

[6] K.Pramod Reddy. BIM for Building Owners and Developers Making a Busi- ness Case for Using BIM on Projects[M]. Canada: John wiley\&Sons, Inc, 2012.

[7] R. Ramponi, B. Blocken. CFD simulation of cross-ventilation for a generic iso- lated building: Impact of computational parameters[J].Building and environ- ment,2012,53(Jul.):34-48.

[8] Brett Steele.O-14 Projection and Reception[M]. London: Architectural Asso- ciation,2012.

[9] Youngsoo Jung, MiheeJoo. Building information modeling (BIM) framework for practical implementation[J]. Automation in construction,2011,20(2):126-133.
[10] I-Tung Yang, Yi-Hung Hsieh. Reliability-based design optimization with dis- crete design variables and non-smooth performance functions: AB-PSO algo- rithm[J].Automation in construction, 2011,20(5):610-619.

[11] Joana Carla Soares Goncalves. Erica Mitie Umakoshi. The Environmental Performance of Tall Buildings[M].London:Earthscan, 2010.

[12] Sumi Lee, Doosam Song. Prediction and evaluation method of wind environ- ment in the early design stage using BIMbased CFD simulation [J]. IOP Conference Series: Materials Science and Engineering, 2010,10(1).

[13] Penttil H, Peter M, Elger D. Evaluating VBE And BIM-Frameworks: A Cost Estimation Case Study and Reflections to Environmental Issues [J]. Helsinki Univer- sity of Technology, Finland, 2009.

[14] Yun Kyu Yi, Ali M. Malkawi. Optimizing building form for energy perfor- mance based on hierarchical geometry relation[J]. Automation in construc- tion,2009,18(6):777-789.

[15] Arno Schlueter, Frank Thesseling. Building Information Model Based Ener- gy/Exergy Performance Assessment In Early Design Stages[J]. Automation in Con- struction,2009,18(2):153-163.

[16] Renaud Vanlande, Christophe Nicolle, Christophe Cruz et al. IFC and build- ing lifecycle management[J].Automation in Construction, 2008,18(1):70-78.

[17] Feng YUAN1, a, Li HAN2, b and Yongheng HU, The BIM 'families' based Environmental Performance Design Research , Advanced Materials Research Vols. 779-780 (2013) pp 1643-1647 